\title{
Angled broad-area semiconductor lasers to emit high output power with good beam quality
}

\author{
Yi-Shin Su ${ }^{\text {a }}$, Chih-Hung Tsai ${ }^{\mathrm{b}}$, Chia-Wei Tsai ${ }^{\mathrm{b}}$, D.P. Tsai ${ }^{\mathrm{b}}$, and Ching-Fuh Lin ${ }^{\mathrm{a}, \mathrm{c}}$ \\ ${ }^{a}$ Institute of Electro-Optical Engineering, National Taiwan University, Taipei, Taiwan, R.O.C. \\ ${ }^{\mathrm{b}}$ Department of Physics, National Taiwan University, Taipei, Taiwan, R.O.C. \\ ${ }^{\mathrm{c}}$ Department of Electrical Engineering and Institute of Electronics Engineering
}

\begin{abstract}
A new type of laser diodes with good beam quality is introduced. The far-field divergence angle can be close to diffraction-limited value. In the new design, the direction of the waveguide on a broad area Fabry-Perot laser diode is tilted at an angle from the facet normal. This design is called "angled broad area laser diode". In this tilted waveguide device, filamentation is not observed. The far-field divergence angle is generally within 5 times the diffraction-limited value. This tilted broad area laser is advantageous over the angled grating DFB laser because the difficulty of matching the grating period with peak gain wavelength is avoided.
\end{abstract}

Keywords: high power laser diode, filamentation, angled broad area laser, angled grating DFB laser, indium phosphide

\section{INTRODUCTION}

Diode Lasers are the type of lasers with the best efficiency. More than 50\% over all power conversion efficiency is common for diode lasers. In comparison, the efficiency of solid state lasers such as $\mathrm{Nd}^{3+}:$ YAG lasers are on the order of $1 \%-10 \% .{ }^{1}$ Current epi-growth technique and processing techniques have enabled the mass production of GaAs-based and InP-based laser-diode chips. The wavelengths of these commercial products covered from $635 \mathrm{~nm}$ to $1600 \mathrm{~nm}$. This range includes the wavelengths of most solid-state lasers. Thus, diode lasers will be an absolutely better choice of light source if efficiency is the only factor considered in lasers. Unfortunately, diode lasers are usually of low power, limited to $<100 \mathrm{~mW}$ mostly.

To make a diode laser emits optical power at thousands of milli-watts, the emitting facet must be large to prevent catastrophic optical damage (COD). ${ }^{2}$ Even with a high COD threshold $\left(10 \mathrm{MW} / \mathrm{cm}^{2}\right)^{3}$, the emitting facet is required to be more than $30 \mu \mathrm{m}$ to make COD threshold three times higher than the optical intensity for 1 watt operation. Thus, a high power diode laser is usually broad and flat. More than $30 \mu \mathrm{m}$ broad emitting facets is necessary for watt scale operation ${ }^{4}$. This reduces the difference of threshold gain between high order modes and fundamental mode, leading to the operation of multiple transverse modes. In addition, the nonlinear interaction between carrier and light will produce filamentation of optical power distribution. All these reasons make high order transverse modes easily excited in broad area Fabry-Perot laser diode and the spatial coherence of emissions on every part of the facets is low.

High power and good beam quality are both desired for many applications such as frequency doubling ${ }^{5}$, ,free space communication $^{6}$, and material processing ${ }^{7}$. To improve the beam quality, approaches such as tapered power amplifiers ${ }^{8}$, antiguided arrays ${ }^{9}$ and angled-grating DFB laser ${ }^{10}$ had been investigated. Although, master oscillator -tapered amplifier systems can provide output in watt scale. Beam filamentation remains to be a problem and trade off between modal gain and beam quality must be made ${ }^{11}$. Angled-grating DFB laser can provide good beam quality. Its best efficiency is lower than a Fabry-Perot LD theoretically. In addition, the mismatch between peak gain wavelength and DFB resonance wavelength defined by grating periods can severely affect the device performance ${ }^{12}$. This imposes a strict requirement on the fabrication of DFB structures. For the same reason, temperature variation can also have bad effect on the device operation due to the shift of the gain profile.

We propose a new type of broad-area laser diodes that are capable of emitting near diffraction-limited beam. These devices do not require the DFB structure, so its fabrication is much simpler. The device geometry will be described, and the device performance will be discussed. 


\section{Device Structure}

Figure 1 is the photograph of such a fabricated laser diode (LD). This device has double channel ridge waveguide etched by reactive ion etch (RIE). The process gases are $\mathrm{CH}_{4}$ and $\mathrm{H}_{2}$. The channel button is 100nm 200nm above the undoped optical confinement layer. Unlike Fabry-Perot LDs, the waveguide direction is not perpendicular to cleaved facets, but tilted at an angle $\theta$. Device parameters such as L(length ), W(width), and $\theta$ (tilt angle) are marked in the figure.

After waveguide is created by RIE, silicon oxide grown by PECVD is used as insulating dielectric. Either wet or dry etch can be used to create the contact window. The contact metal is Ti/Pt/Au on p-side and AuGe/Ni/Au on n-side.

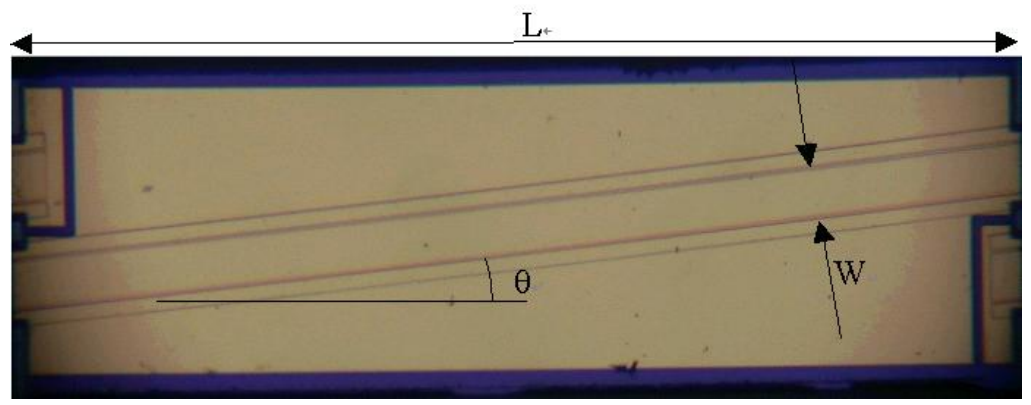

Figure 1. Photo of the device after cleaving

\section{Device Characteristics}

Figure 2 is the L-I curve of this device from a single facet. Unlike tilted waveguide devices with small waveguide width, this device still behaves like a laser diode. The threshold of this device is $4.2 \mathrm{~A}$ without facet coating and external feedback. The slope efficiency after threshold is $0.053 \mathrm{~mW} / \mathrm{mA}$. At $10 \mathrm{~A}$, this device provides $300 \mathrm{~mW}$ optical output from each facet. Before threshold, the emission is mainly contributed by spontaneous emission. The spectrum is very broad with $94 \mathrm{~nm}$ FWHM at 2A. As the current is increased to near threshold level, the spectrum FWHM decreases due to stimulated emission, but the FWHM is still $64 \mathrm{~nm}$ broad. After threshold, the spectrum width is less than $12 \mathrm{~nm}$. The wavelength of oscillation is $1611 \mathrm{~nm}$ (Figure 3). Before threshold, the emitting area on the facet is broad and covers the whole device facet (Figure 4). When the injection level is close to threshold, the near field pattern narrows a little, and a small peak can be observed on the position of the waveguide (Figure 5). When the current is increased above threshold level, most of the emitted light concentrates around the waveguide, but the area of emission is still broader than the waveguide width. The near field of this device is smooth and asymmetric. Figure 6 and Figure 7 show the near field measured at 6A. Unlike broad area laser diodes with waveguide parallel to facet normal, no filamentation is observed on the near field patterns. The waveguide width is $100 \mu \mathrm{m}$. At side A, the near-field FWHM is $138 \mu \mathrm{m}$. At side B, the near-field FWHM is $143 \mu \mathrm{m}$. The corresponding diffraction limited far-field FWHM is $0.8^{\circ}$. At low injection current density(below threshold level), the far field pattern is mainly contributed by spontaneous emission as shown in Figure 8. When the injection current is close to threshold level, a small peak is observable at the direction of facet normal(Figure 9). When the current is increased above threshold, the laser emission points at the direction of facet normal. A secondary peak can be observed to grow as laser emission at facet normal increase. Thus, the measured far field at $6 \mathrm{~A}$ has two peaks, as shown in Figure 10 and Figure 11. In Figure 10, the major peak is centered at facet normal and has $3.7^{\circ}$ FWHM, and the second peak is $49^{\circ}$ away from facet normal. In Figure 11, the second peak is $52^{\circ}$ away from facet normal. In both Figure 10 and Figure 11, the second peaks and the waveguide directions are at the same side relative to the facet normal. Both peaks exist only at currents higher than threshold level. From the near field and far field patterns at 2A, no amplified spontaneous emissions are observed. The amplified spontaneous emissions should concentrate on the waveguide in the near-field pattern, and they should be observed at $23^{\circ}$ away from facet normal. This fact indicates that the threshold gain of the laser oscillation is not very high. Thus, this tilted waveguide structure does not suppress the oscillation modes much. 


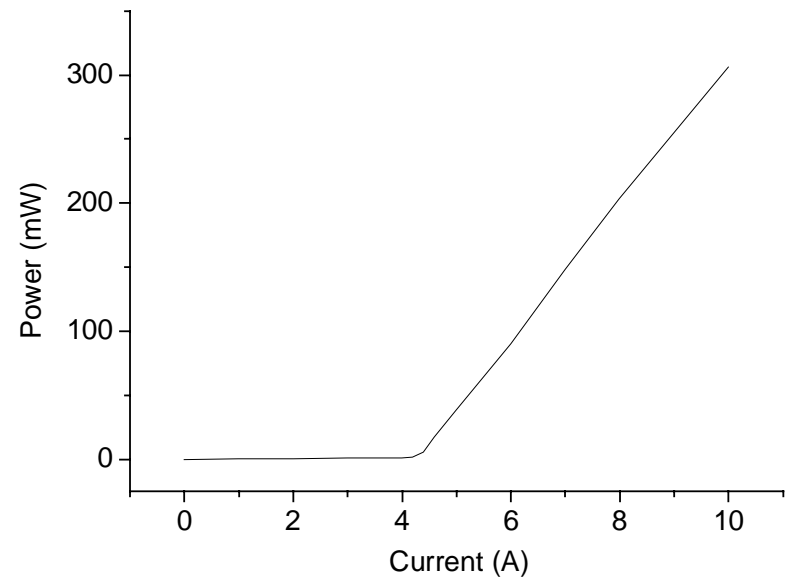

Figure 2 The L-I curve of the device with $\mathrm{L}=1600 \mu \mathrm{m}$ and $\mathrm{W}=100 \mu \mathrm{m}$, facet A.

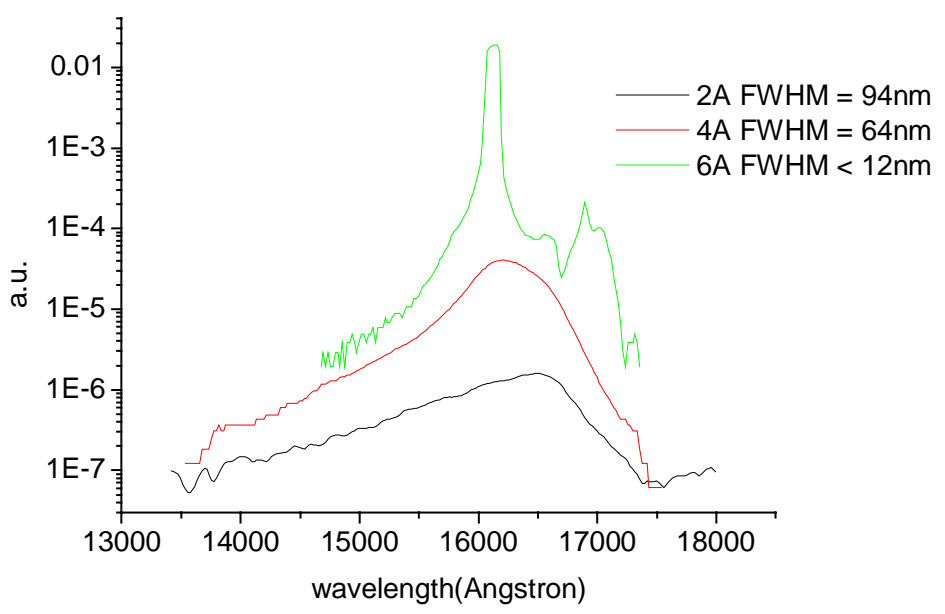

Figure 3 Spectrum of this device at different current

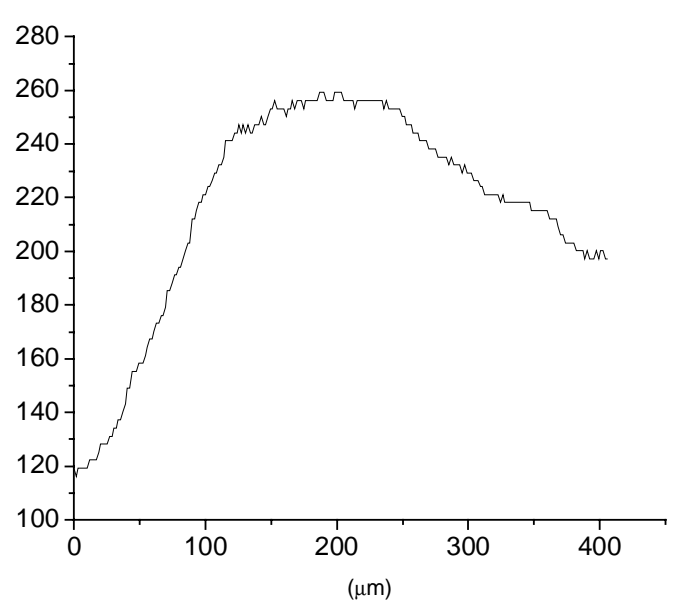

Figure 4 The near field of the angled broad-area device at 2A, facet A. 


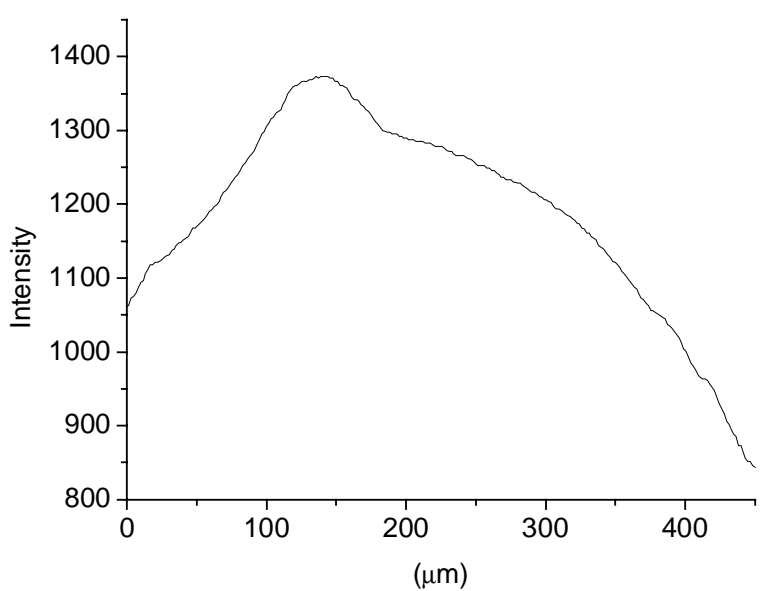

Figure 5 The near field of the angled broad-area device at $4 \mathrm{~A}$, facet A.

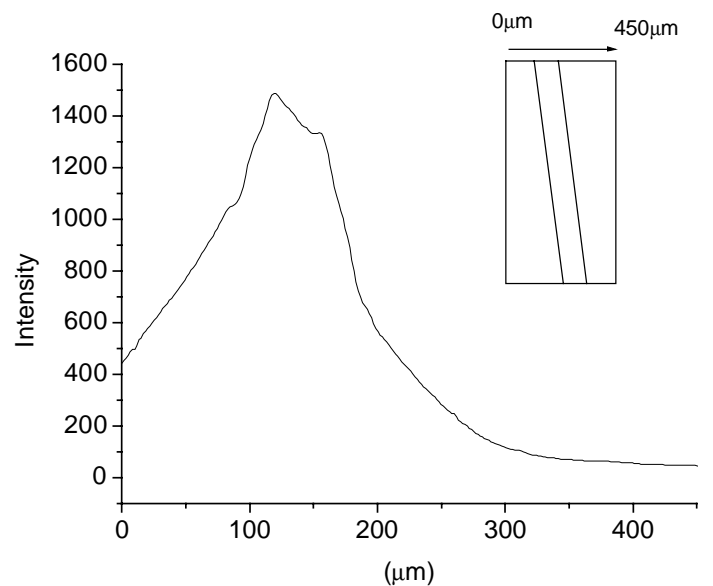

Figure 6 The near field of the angled broad-area device at 6A, facet A.

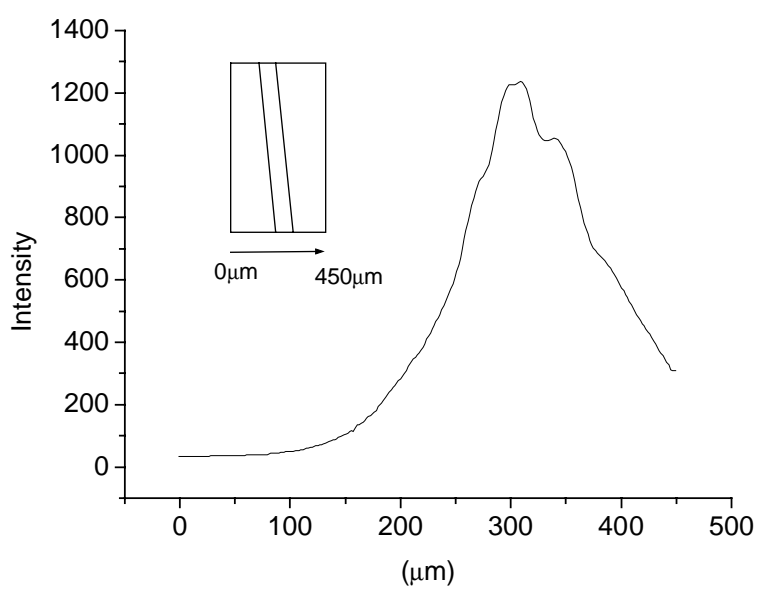

Figure 7 The near field of the angled broad-area device at 6A, facet B. 


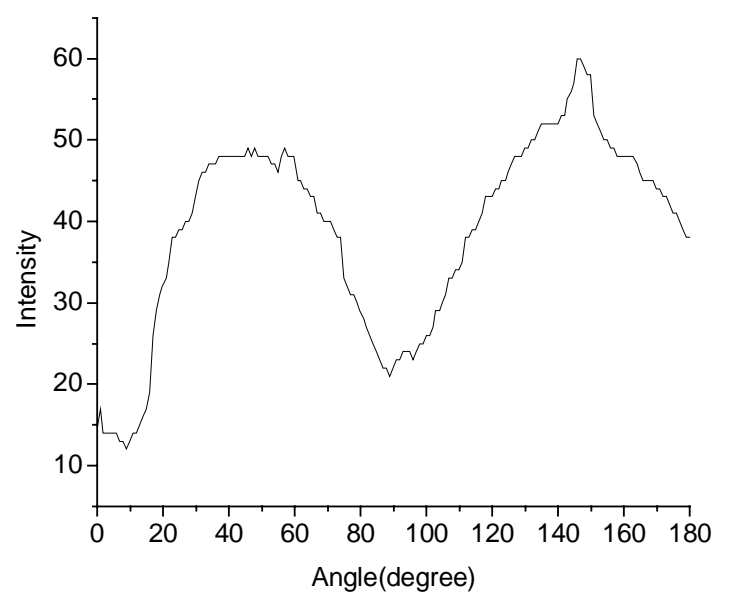

Figure 8 The measured far field of side $\mathrm{A}$ at $2 \mathrm{~A}$.

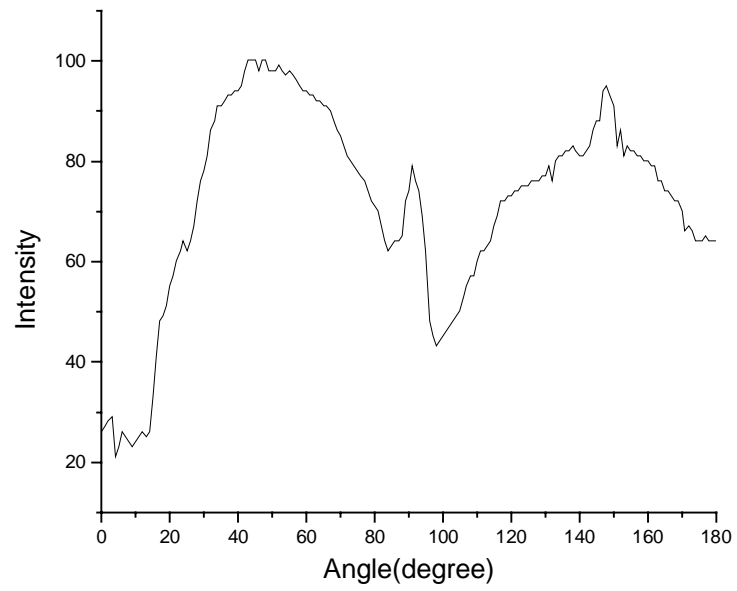

Figure 9 The measured far field of side $\mathrm{A}$ at $6 \mathrm{~A}$.

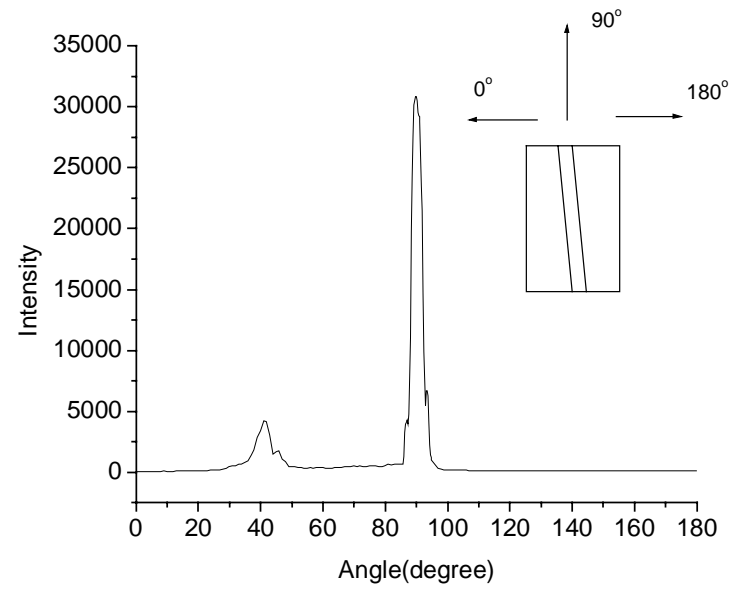

Figure 10 The measured far field of side $\mathrm{A}$ at $6 \mathrm{~A}$. 


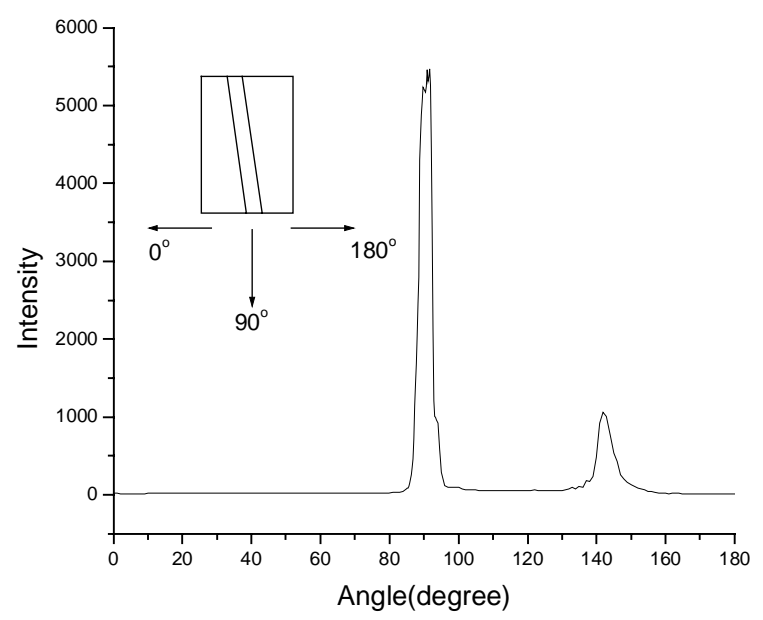

Figure 11 The measured far field of side B at 6A.

\section{Modes of Oscillation}

There are two possible types of oscillations that can occur in an angled broad area laser diode (Fig. 12). One is the straight path oscillation, which penetrates out off the waveguide as shown in figure 12(a). For this oscillation to occur, the absorbing materials in the unpumped region must be saturated. Thus, the L-I curve will have a jump at onset of oscillation as shown in Figure 13. The first oscillation mode occurs at 822mA, and the L-I curve has a step at this current. There is another step at $952 \mathrm{~mA}$, indicating the onset of another oscillation mode. The existence of absorbing region makes the efficiency low. When the number of oscillating modes is not much, these modes can be separated from far-field pattern. As shown in Figure 14, the far-field pattern has only one peak at $854 \mathrm{~mA}$ and multiple peaks at $994 \mathrm{~mA}$. If this is the path of oscillation, which occurs in the devices mentioned in the previous section, we should observe clear kinks on the L-I curve. In addition, the oscillation mode, which penetrates out off the waveguide, should be observed on

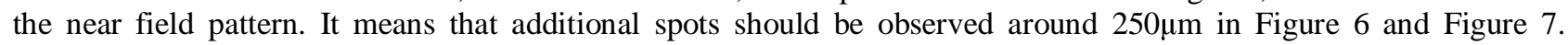
However, these extra spots are not observed on the near field patterns, and kinks are not observed in the L-I curve of this device. Thus, it is less possible that this device oscillates in straight mode.

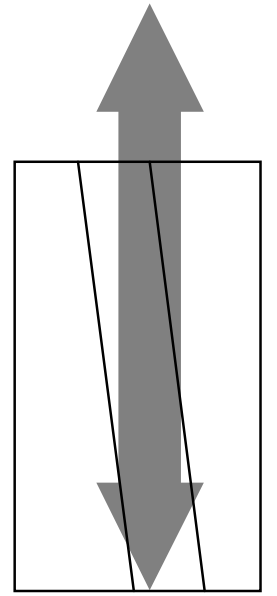

(a)

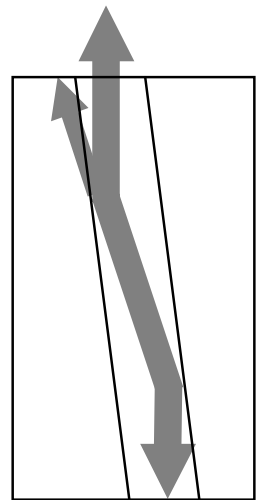

(b)

Figure 12 Two possible oscillation path of an angled broad area laser diode. 


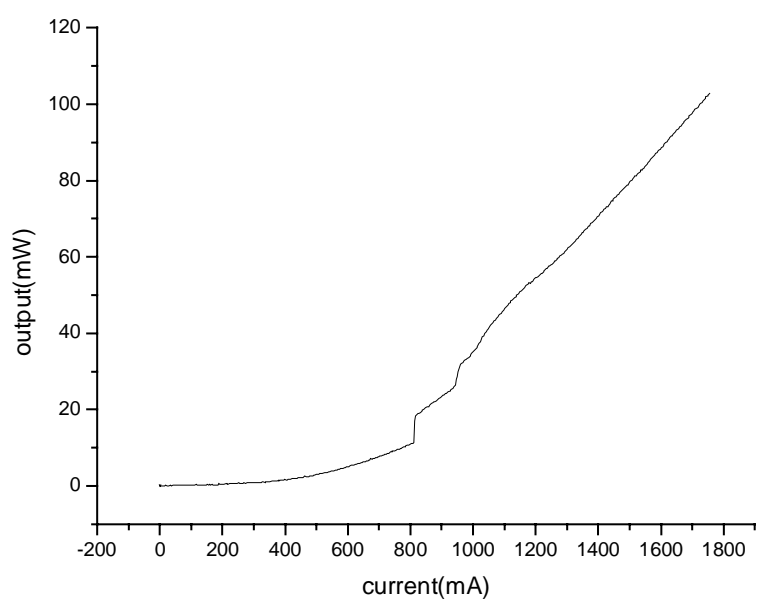

Figure 13 The L-I curve of a device with $\mathrm{L}=1000 \mu \mathrm{m}$ and $\mathrm{W}=50 \mu \mathrm{m}$.

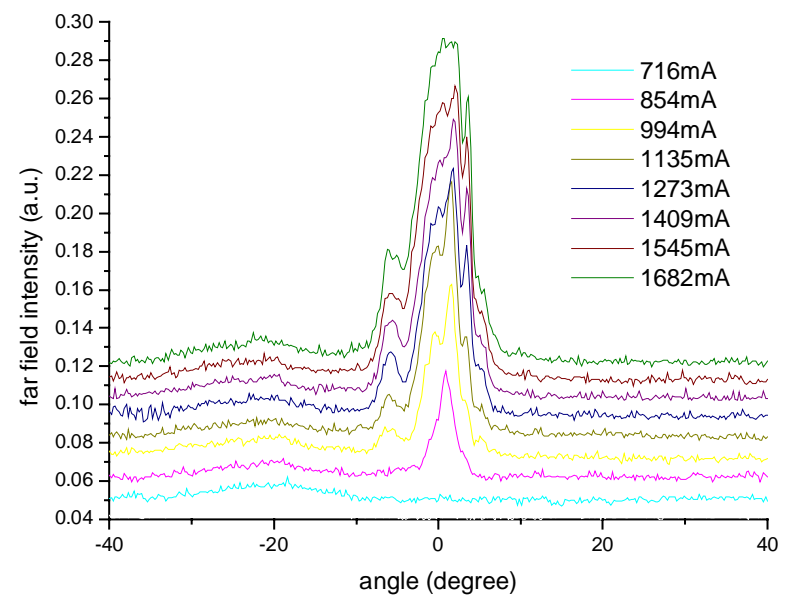

Figure 14 The corresponding far field of the device in figure 13.

Another possible oscillation path is a zigzag optical path (Fig. 12(b)), which is similar to that occurs in angled-grating DFB lasers. For such a mode to oscillate, the cavity length should be chosen in a way such that the following equation is satisfied. This is the condition for the light propagating in the waveguide to complete exactly one zigzag cycle, when it propagates from one facet to another facet and be back again. For $\mathrm{W}=100 \mu \mathrm{m}$ and $\theta=7^{\circ}$, the optimum L is near $1600 \mu \mathrm{m}$. Because reflection from waveguide edge may not be very efficient, it is also reasonable that the slope efficiency of this device is quiet low. If the device length has some deviation from this optima value, there will be some none ideal effect on the device. Figure 15 shows the resulting oscillation path for a device longer than the optima length. In this figure, part of the light must propagate through absorbing region before reflected back by facet at the right hand side. This absorbing region can also produce kinks on the L-I curve. This effect may be less observable because the area of absorbing region is smaller than the straight oscillation path. In addition, a part of the light may be reflected by waveguide edge before it hits the facet and leaves the device in an angle different from facet normal. Thus, a second beam may be observed on the far-field pattern. In addition, this second beam should leave the device at an angle of $53^{\circ} \sim 57^{\circ}$ away from facet normal according to Snell's law because its propagation direction is $14^{\circ}$ away from 
facet normal in the waveguide. This angle is the same as that observed in Figure 10 and 11. A similar effect can happen in case of straight oscillation path (Figure 16), but the near field patterns of the straight and zigzag oscillation modes are different. In the case of zigzag oscillation path, the near field should concentrate around the waveguide. Even when the device length has some deviation from ideal value, the near field should still concentrate around the waveguide. This is the case observed in this experiment. Thus, the device observed in this experiment should oscillate in the zigzag mode.

$$
L=2 W \cos (\theta) / \tan (\theta)
$$

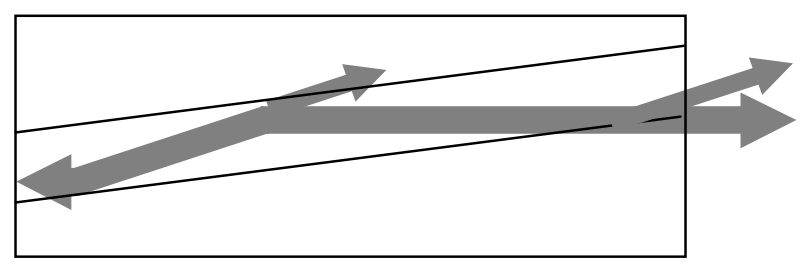

Figure 15 The zigzag oscillation path for a device which is too long.

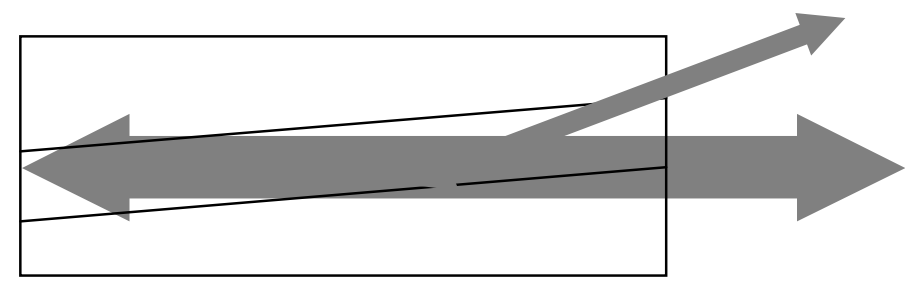

Figure. 16 The origin of the second far-field peak in straight oscillation mode.

\section{Conclusion}

In this work, we introduce a new type of laser structures. The waveguide of diode lasers using this type of structure can be broad and long, but no filamentation occurred in the device. The mode of oscillations in this device should be of zigzag type judging from near-field observation. This laser can provide output with near diffraction limited farfied divergence angle. Under proper condition, this laser can give diffraction-limited beam. In the average case, this laser can give far-field FWHM within five times of diffraction-limited value. In addition, these tilted broad area lasers are easy to fabricate. No DFB or photonic crystal structures are required. Thus, they are suitable for mass production.

\section{References}

1.Amnon Yariv “Optical Electronics in Modern Communications”, Chap. 7, Oxford, New York, 1997

2. Govind P. Agrawal and Niloy K. Dutta "Semiconductor Lasers" Chap. 14, Van Nostrand Reinhold, New York, 1993

3. M. Fukuda, "Reliability and degradation of semiconductor lasers and LEDs." Boston, MA: Artech, 1991

4. Emanuel, M.A.; Carlson, N.W.; Skidmore, J.A.” High-efficiency AlGaAs-based laser diode at 808 nm with large 
transverse spot size” Photonics Technology Letters, IEEE, Volume: 8 Issue: 10 , Oct., p.p. 1291 -1293, 1996

5. B. Beier, D. Woll, K.-J. Boller, R. Wallenstein: "Second-harmonic generation of the output of an AlGaAs diode oscillator-ampli.er system in critically phase matched LiB3O5 and b-BaB2O4”, Appl. Phys. Lett. 71, p.p. 1 - 3, 1997

6. S. G. Lambert, W. L. Casey: "Laser Communication in Space” Boston, MA: Artech, 1995

7. P. Loosen, H.-G. Treusch, C. R. Haas, U. Gardenier, M. Weck, V. Sinnho., St. Kasperowsky, R. vor dem Esche "High-power diode lasers and their direct industrial applications", SPIE Proc. 2382, p.p. 78 - 88, 1995

8. J. N. Walpole, "Semiconductor amplifiers and lasers with tapered gain regions." Opt. Quantum. Electron. Vol.28, $623-645,1996$

9. C. Smudzinski, D. Botez, L. J. Mawst, A. Bhattacharya, M. Nesnidal, R. F. Nabiev, "Three-core arrow-type diode laser: novel high-power, single-mode device, and effective master oscillator for .flared antiguided MOPAs." , IEEE J. Select.Topics Quant. Electron. Vol.1, 129-137 ,1995

10. S. D. de Mars, K. M. Dzurko, R. J. Lang, D. F. Welch, D. R. Scifres,A. Hardy, “ Angled grating distributed-feedback laser with 1W single-mode, diffraction-limited output at $980 \mathrm{~nm}$, Techn. Digest CLEO 96 CTuC2, pp. $77-78,1996$

11. M. Mikulla, et al, "Improved beam quality for high-power tapered diode lasers with LMG epitaxial layer structures", SPIE Proc. 3284, 72 - 79, 1998

12. I. Vurgaftman, W. W. Bewley, R. E. Bartolo, C. L. Felix, M. J. Jurkovic, J. R. Meyer, M. J. Yang H. Lee and R. U. Martinelli "Far-field characteristics of mid-infrared angled-grating distributed feedback lasers" J. Appl. Phys. Vol. 88, Num 12, p. 6997, Dec. 2000 